\title{
DIVERSITÉ DES COMPOSÉS EN COUCH, DE LEURS ÉQUIVALENTS ET DE LEURS COMMENTAIRES
}

\author{
JEAN-FRANÇOIS SABLAYROLLES', ANNA BOBIŃSKA, \\ JOHN HUMBLEY
}

\begin{abstract}
Diversity of compounds including couch, their equivalents and comments

The aim of this article is to study the diversity of the forms taken by compounds in which the loan couch appears in French and Polish, taking into account the diversity of their equivalents and the comments made about them. Two loans are particularly well represented: couch surfing and couch potato and two others, couch gag and couch promotion, both less frequent and less widespread. A large number of other cases are found in the corpus which also include couch, many of which are hapaxes. Analysis of the results in both languages but more particularly in French reveals that the more frequent the loanword is, the greater the variety of both native equivalents proposed and comments on both the loan and the appropriate translation.
\end{abstract}

Keywords : Loanword, equivalent, explanation, typology

Mots-clés : Emprunt, équivalent, glose, typologie

\section{Introduction}

Parmi les emprunts à l'anglo-américain de ces dernières décennies, ceux qui comprennent le formant couch ont la particularité d'être attestés sur une assez longue durée. Celui qui fut le premier de la série, couch potato, a servi de modèle à un grand nombre d'autres composés, créés au fil du temps, dont beaucoup se sont diffusés à divers degrés en français et en polonais. Ces emprunts ont aussi donné lieu à un grand nombre d'équivalents autochtones concurrents et à de nombreux commentaires.

L'objectif de cet article est de rendre compte de la pénétration de cette famille lexicale dans les deux langues de l'étude, d'apprécier son assimilation mais surtout d'examiner de près les stratégies de nomination des nombreux équivalents qui l'accompagnent le plus souvent sous forme de glose. Par rapport au chapitre sur binge $e^{2}$, qui se focalise sur

1 Jean-François Sablayrolles (25.04.1951 - 11.12.2020) n'a pas eu le temps de terminer cet article mais c'est lui qui a effectué toutes les recherches dans les archives d'Europresse et qui a esquissé le plan de l'article ainsi que les analyses pour le français.

2 Voir l'article «Diversité des liens entre un emprunt et ses équivalents - le cas de binge drinking» dans ce volume. 
les segments glosateurs (Steuckardt et Niklas-Salminen 2005) qui introduisent un équivalent, la présente étude se donne pour but de faire ressortir les stratégies de nomination employées dans les deux langues.

Le partage dans les gloses entre les équivalents et les commentaires n'est pas toujours net. Il y a sans doute une sorte de continuum entre des traductions mot-à-mot (souvent données comme telles avec des marques comme « littéralement»), des calques plus ou moins fidèles, des synthèses néologiques puis des explications qui ne sont pas à proprement parler des équivalents mais qui restent assez proches de la source de langue anglaise. D'autres reformulations sont de type encyclopédique tandis que d'autres encore sont des jugements subjectifs du scripteur portés sur la réalité dénommée.

\section{Méthode}

C'est le français, en tant que langue-pivot de cette série d'études, qui fournit le point de départ du dépouillement. Un premier corpus a été constitué à partir d'une requête couch portant sur les 500 occurrences de ce mot disponibles entre début 2012 et début avril 2020 sur les pages françaises d'Europresse, base de données de la presse ${ }^{3}$, ce qui livre, après vérification, environ 178 occurrences pertinentes. Depuis 1944, il y en a 8229 en tout (requête d'avril 2020), mais la plupart sont hors sujet, en particulier du fait de la présence de nombreuses petites annonces de locations, de caravanes, etc. indiquant le nombre de couchages avec la forme accourcie couch. Les différents composés en couch relevés lors de ces premières interrogations ont fait l'objet de requêtes sur la totalité des archives des pages françaises disponibles sur Europresse, soit depuis 1944. Des sondages rapides à l'aide du moteur de recherche Google (pages françaises) ont également été effectués. Ils permettent de comparer, dans le meilleur des cas, des ordres de grandeur de diffusion et de constater, à coup sûr, une faible diffusion quand peu de résultats s'affichent.

Pour le polonais, le corpus a été établi à partir de trois sources. Premièrement, la recherche a été effectuée sur deux corpus dans Sketch Engine ${ }^{4}$ - Polish Web 2012 et Timestamped JSI web corpus 2014-2020. Ensuite, des requêtes sur le moteur de recherche Google (pages polonaises) ont été réalisées. Enfin, les pages de Frazeo ${ }^{5}$, moteur de recherche et de comparaison d'actualités du monde entier, compilées par les portails d'information polonais à partir de diverses sources, ont été consultées.

Par ordre alphabétique ont été relevés et sont examinés en français et en polonais : couch air, couch commander, couch compagnie, couch dances, couch detective, couch gag, couch gay, couch news, couch potato/es, couch surfer (euse) sing et pl, couch surfing, (avec ou sans trait d'union pour ces derniers mots), ainsi que casting couch. Deux autres composés ont été relevés dans le corpus français Timestamped JSI 2014-2020 de Sketch Engine : couch battle et couch coop platformer; ils sont également pris en compte.

http://www.europresse.com/, consulté le 26. 03. 2021.

4 https://www.sketchengine.eu/, consulté le 26. 03. 2021. Pour en savoir plus sur les corpus de Sketch Engine, voir Bušta, Herman (2017).

5 http://frazeo.pl/, consulté le 26. 03. 2021. 
La plupart de ces composés sont peu fréquents mais ils sont souvent accompagnés d'un commentaire et d'une proposition d'équivalent. Quatre séries d'inégal développement apparaissent en effet, dont les premières seront approfondies :

1. couch potato et couch surf (er/ing), qui sont abondamment présents dans les deux langues;

2. une série plus limitée : couch gag (toujours lié aux Simpson), et casting couch;

3. les séries très peu développées ;

4. les hapax.

\section{Foisonnement néologique des équivalents}

Couch surfing et couch potato sont abondamment représentés dans les deux langues tout comme leurs équivalents et leurs gloses, qui font l'objet de l'analyse ci-après.

\section{1 couch surf- en français}

Wiktionnaire français donne la définition suivante de couch surfing : « Mode de voyage consistant à choisir son hébergement gratuit sur Internet ${ }^{6} »$.

Wikipédia fournit davantage de détails sur son origine :

En 1999, lors d'un voyage en Islande, le programmeur américain Casey Fenton contacte par liste de diffusion des étudiants de l'université de Reykjavik pour demander à être gracieusement hébergé, avec l'espoir de pouvoir rencontrer des habitants de la région. Le nombre impressionnant de réponses positives le convainc qu'il existe une communauté de personnes pensant autrement le voyage, plus centrée sur l'humain. Au retour de son voyage lui vient l'idée de créer un site web pour mettre en relation les membres de cette communauté. Il enregistre le nom de domaine couchsurfing.org le 13 juin 1999. Ce n'est que quelques années plus tard, le 2 avril 2003 qu'est fondé l'organisme sans but lucratif Couchsurfing International Inc. ${ }^{7}$

En anglais, couch surfing est très probablement un néologisme associé à une métaphore secondaire, le modèle étant channel surfing dans le sens de passer d'une chaîne de télévision à une autre (connu en français sous la forme du faux anglicisme zapping), comme le surfeur passe d'une vague à l'autre. Il est plus souvent question dans le corpus de celui qui accueille que de celui qui loge chez autrui, alors qu'on aurait pu s'attendre à l'inverse : celui qui passe ou surfe d'un canapé à l'autre est en effet plutôt l'hébergé que l'hébergeur. Cela vient peut-être du fait que sur la toile, ce sont les hébergeurs qui sont plus présents, avec des sites où ils proposent leur canapé, alors que ce ne sont pas ceux qui cherchent à se faire héberger qui passent des petites annonces (même si, comme on l'a vu supra, c'est un tel appel qui a été à l'origine de la création du site avant son antonomase en nom commun).

https://fr.wiktionary.org/wiki/couch_surfing, consulté le 26. 03. 2021.

https://fr.wikipedia.org/wiki/CouchSurfing, consulté le 26. 03. 2021. 
Comme le fait remarquer Jacquet-Pfau (2018), couch surfing fait partie des manifestations de l'économie du partage et figure parmi les pratiques emblématiques de cette nouvelle orientation de la société qui a son origine aux États-Unis, au même titre que les phénomènes de crowd- (crowdsourcing, crowdlending, etc.), d'une part, et de co- (coworking) de l'autre. Comme l'indique la citation de Wikipédia, l'origine de cet emprunt est bien documentée : Couchsurfing est le nom d'une entreprise américaine active dans cette économie du partage, qui met en rapport des particuliers qui cherchent et qui proposent un logement temporaire sans frais (un canapé pour y dormir). Il est désormais employé en tant que nom commun. Ce processus relève de l'antonomase, appelée aussi déonomastique (Buchi 1993). Pour les juristes spécialisés dans les noms de marques, il s'agit d'une modification de statut juridique : ils parlent d'une déchéance (Tonti 2020). En effet, un nom de marque qui devient un nom commun ne peut plus être protégé en tant que tel.

\subsection{1 couch surfing}

D'un point de vue statistique, on a relevé 275 occurrences en français dans Europresse du 08. 03. 2004 au 28. 02. 2020 (288, mais 13 répétitions). C'est aussi le nom du réseau international, du site et ou de documentaire d'un voyage en couch surfing dans 53 occurrences. Ce sont donc plutôt 222 occurrences qui sont réellement pertinentes. Les flux ont néanmoins été établis sur les 275 et pas les 222 :

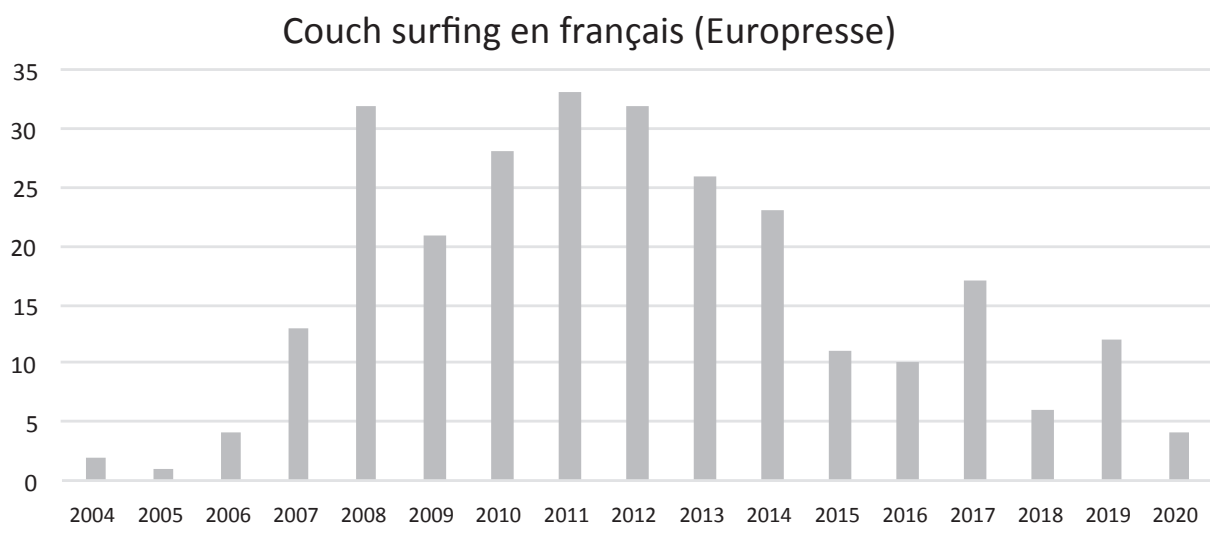

Graphique 1 : Occurences de couch surfing par année dans Europresse

Sauf oubli, 65 emplois sont mis entre guillemets, ce qui représente à peine moins d'un quart de l'ensemble (23,64\%). 80 occurrences sont accompagnées d'une explication, plus ou moins précise et sous différentes formes, ce qui représente $29 \%$ de la totalité.

Des équivalents de l'emprunt sont présents dans le tableau 1 ci-dessous pour 29 occurrences $(10,45 \%)$ avec 10 formulations différentes (à droite figure le nombre d'occurrences dans Europresse) : 
Tableau 1 : Équivalents de couch surfing dans le corpus français

\begin{tabular}{|l|c|l|}
\hline $\begin{array}{c}\text { couch surfing - équivalents } \\
\text { présents dans le corpus }\end{array}$ & $\begin{array}{c}\text { nombre d'emplois } \\
\text { de l'équivalent } \\
\text { dans le corpus }\end{array}$ & $\begin{array}{c}\text { nombre d'occurrences de l'équivalent dans } \\
\text { Europresse }\end{array}$ \\
\hline nuit sur canapé & 1 & seul emploi \\
\hline hébergement gracieux & 1 & 66 emplois, mais pas tous du couch surfing \\
\hline hébergement temporaire & 1 & 8 637, mais peu sont du couch surfing \\
\hline hébergement sur le canapé & 1 & 4 , dont tous sont du couch surfing, présent 3 fois \\
\hline tourisme sofa & 1 & seul emploi \\
\hline canapé en ligne & 1 & $\begin{array}{l}\text { seul emploi pertinent : sur les } 8 \text { affichés, } 7 \text { de } \\
\text { vente }\end{array}$ \\
\hline hébergement sur le canapé & 1 & 3 dont 2 avec couch surfing et 1 sans \\
\hline passer d'un canapé à l'autre & 2 & 41 très souvent en lien avec couch surfing \\
\hline $\begin{array}{l}\text { prêt de / se faire prêter } \\
\text { un canapé }\end{array}$ & $5 ?$ & 5 ? très souvent avec couch surfing \\
\hline surf(ing) / surfer sur canapé & 15 & 43 souvent en lien avec couch surfing \\
\hline
\end{tabular}

On relève beaucoup d'explications, même encore maintenant, ce qui laisse supposer que la pratique, même si elle se répand, reste encore peu connue du grand public.

\subsection{2 couch surfe $(u) r$}

On relève pour couch surfe $(u) r$ masc. et fém, sing. et pl. 70 occurrences différentes, toutes formes fléchies confondues du 8/03/2004, l'année de l'apparition aux États-Unis d'après certains sites, au 01. 07. 2019 (donc sur une période de 15 ans). Seulement neuf sont mis entre guillemets, ce qui représente le faible pourcentage de $12,85 \%$. Les traductions sont encore moins nombreuses : on n'en trouve que $2(2,85 \%)$. Les explications ne sont pas non plus très abondantes, puisqu'on n'en relève que 10 (14,28\%). Ces trois résultats contrastent un peu avec ceux de couch surfing qui sont plus élevés. Il s'agit quasiment toujours de l'hébergeur et très rarement de l'hébergé, comme cela apparaît dans les sites suivants : hospitalityclub.org créé en août 2000 par l'Allemand Veit Kuhne (311 000 membres en 2007) et couchsurfing.com créé en janvier 2003 par l'Américain Casey Fenton (265 000 membres en 2007) (voir aussi Jacquet-Pfau 2018 : 188).

\section{2 couch surf- en polonais}

\subsection{1 couchsurfing}

Pour le polonais, les premières attestations du mot couchsurfing sur Internet remontent à l'année 2006, mais il faut souligner qu'elles sont réellement peu nombreuses. Il est également difficile de fixer la date de la première apparition de cet emprunt en polonais, en tenant compte du fait que, dans la plupart des cas, il s'agit des sites ou des articles 
rédigés en anglais. Actuellement, la recherche sur le moteur Google affiche environ 6340000 résultats pour la lexie en question. Cependant, il convient de préciser que ce nombre englobe aussi bien les pages en anglais et en polonais que le nom de l'entreprise commerciale et son site. En revanche, le moteur Frazeo propose 56 occurrences pour couchsurfing, y compris celles qui apparaissent dans la presse en ligne, la première de l'année 2009. La plupart d'entre elles sont accompagnées d'une explication ou d'un court commentaire :

(1pl) Couchsurfing to dosłownie „surfowanie po kanapach”, czyli darmowe korzystanie z miejsca noclegowego. Jest ono możliwe dzięki portalowi społecznościowemu Couchsurfing. org, który zrzesza couchsurferów z całego świata, umożliwiając im „wymianę gościnności“" [Couchsurfing signifie littéralement „surfer sur les canapés“, soit l'utilisation gratuite de l'hébergement. Elle est rendue possible par le site de réseau social Couchsurfing.org, qui réunit des couchsurfeurs du monde entier, leur permettant « d'échanger l'hospitalité ».]

La recherche dans les deux corpus polonais de Sketch Engine s'avère un peu plus fructueuse. On note 780 résultats pour couchsurfing et 147 pour couch surfing dans le corpus Polish Web 2012 et, ce qui est encore plus curieux, uniquement un résultat pour couch surfing et 41 résultats pour couchsurfing dans le corpus postérieur, à savoir Timestamped JSI web corpus 2014-2020. Tous les contextes dans lesquels ces mots apparaissent gardent un lien étroit soit avec la façon de voyager et de visiter le monde déjà décrite, soit avec une communauté cosmopolite centrée autour du site à l'origine de ce phénomène :

(2pl) Obok autostopu, carpoolingu i zamiany domami, Couchsurfing uważany jest za jeden z najlepszych sposobów na tanie podróżowanie. Jednak tu nie chodzi tylko o darmowy nocleg. Najważniejsze jest spotkanie, interakcja, do której dochodzi między przedstawicielami innych, czasem bardzo różnych kultur. Couchsurfing to również - poza możliwością znalezienia noclegu - świetne źródło wiedzy o odwiedzanych miejscach ${ }^{9}$.

[Avec l'auto-stop, le covoiturage et l'échange de maisons, le couchsurfing est considéré comme l'un des meilleurs moyens de voyager à moindre coût. Toutefois, il ne s'agit pas seulement d'un logement gratuit. Le plus important est la rencontre, l'interaction qui a lieu entre des représentants d'autres cultures, parfois très différentes. Le couchsurfing est aussi - outre la possibilité de trouver un logement - une grande source de connaissances sur les lieux visités.]

Sans surprise, on relève en polonais de nombreuses modifications morphologiques, contrairement au français, sous la forme des suffixes désinentiels. On constate par exemple l'emploi des formes déclinées. Il s'agit le plus souvent des substantifs masculins et féminins, mais aussi des adjectifs ante- ou postposés, accordés en genre et en nombre avec le nom auquel ils se rapportent :

- idea ('idée du') couchsurfingu

- przygoda z couchsurfingiem ('aventure avec')

8 http://dziennikturystyczny.pl/2014/06/couchsurfing/, consulté le 26. 03. 2021.

9 https://www.ethnopassion.pl/2017/12/DziewczynskiCouchSurfing.html, consulté le 26. 03. 2021. 
- doświadczenia ('experiences') couchsurfingowe

- osobowość ('personnalité) couchsurfingowa

- couchsurfingowi przyjaciele ('amis')

- ruch ('mouvement') couchsurfingowy

En ce qui concerne les équivalents polonais du couchsurfing, il semble que surtout deux mots se soit relativement bien ancrés dans la langue, en particulier dans la blogosphère et les forums en ligne : surfująca kanapa ('canapé qui surfe'), surfowanie po kanapie/po kanapach ('surf sur le canapé/les canapés'). En outre, il existe deux unités couchsurfingowanie et kanapasurfing, dont principal intérêt réside dans le fait que seulement l'un des éléments composants est traduit en polonais. Toutes ces formes sont néanmoins un peu moins répandues que le mot anglais. En plus, elles accompagnent très souvent ce dernier, servant ainsi d'explication :

(3pl) Czy słyszeliście o couchsurfingu? Surfująca kanapa to amerykański pomysł, zgodnie z którym, jeśli masz wolną przestrzeń w swoim domu, możesz ją udostępnić podróżnikom i turystom ${ }^{10}$.

[Avez-vous entendu parler du couchsurfing ? Le canapé qui surfe est une idée américaine selon laquelle si vous disposez d'un espace libre dans votre maison, vous pouvez le partager avec les voyageurs et les touristes.]

Finalement, le nombre de résultats pour chaque équivalent se répartit comme indiqué dans le tableau 2 ci-dessous :

Tableau 2 : Dérivés polonais de couch surf

\begin{tabular}{|l|c|c|c|r|}
\hline & $\begin{array}{c}\text { Polish Web 2012 } \\
\text { Sketch Engine }\end{array}$ & $\begin{array}{c}\text { Timestamped JSI web } \\
\text { corpus 2014-2020 Polish } \\
\text { Sketch Engine }\end{array}$ & Frazeo & Google \\
\hline couchsurfowanie & 0 & 0 & 0 & 4 \\
\hline kanapasurfing & 0 & 0 & 0 & 262000 \\
\hline surfująca kanapa & 0 & 0 & 0 & 3080 \\
\hline surfowanie po kanapie & 2 & 0 & 5 & 58300 \\
\hline surfowanie po kanapach & 13 & 0 & 6 & 39600 \\
\hline
\end{tabular}

\subsection{2 couchsurfer (masculin), couchsurferka (féminin)}

Employés au singulier (couchsurfer, couchsurferka) et au pluriel (couchsurferzy, couchsurferki), parfois aussi déclinés, selon le contexte, ces deux noms indiquent premièrement celui et celle qui réalise un voyage de ce type, ensuite celui et celle qui prête son canapé aux voyageurs de passage. Le polonais semble ainsi plus proche de l'anglais que le français. Il existe également un autre suffixe dérivationnel s'appliquant au nom d'agent, et

10 http://www.kasiavictor.com/pl/couchsurfing-dla-poczatkujacych/, consulté le 26. 03. 2021. 
par conséquent, un substantif formé avec - owiec : couchsurfingowiec. Le féminin pour ce mot n'est pas attesté dans notre corpus, bien que le suffixe -owczyni soit remarquablement productif en polonais ces derniers temps, ceci s'explique sans doute par les difficultés de prononciation.

Le nombre des résultats pour chaque forme, selon différentes sources, se répartit comme indiqué dans le tableau 3 ci-dessous :

Tableau 3 : Dérivés polonais de couch surf

\begin{tabular}{|l|c|c|c|r|}
\hline & $\begin{array}{c}\text { Polish Web 2012 } \\
\text { Sketch Engine }\end{array}$ & $\begin{array}{c}\text { Timestamped JSI web } \\
\text { corpus 2014-2020 Polish } \\
\text { Sketch Engine }\end{array}$ & Frazeo & Google \\
\hline couchsurfer & 42 & 0 & 1 & 481000 \\
\hline couchsurferzy & 49 & 1 & 3 & 1140000 \\
\hline couchserferka & 9 & 1 & 10 & 1300 \\
\hline couchsurferki & $7^{*}$ & 0 & 0 & $2030^{*}$ \\
\hline couchsurfingowiec & 2 & 0 & 0 & 224 \\
\hline couchsurfingowcy & 3 & 0 & 0 & 218 \\
\hline
\end{tabular}

* pour cette entrée, les résultats s'avèrent peu pertinents - sans contexte, ils peuvent s'appliquer à la fois au nom féminin pluriel et au génitif pour le singulier.

\section{3 couch potato}

\subsection{1 couch potato en français}

Wiktionnaire donne la définition suivante de couch potato : "Personne qui passe beaucoup de temps assis ou couché, souvent à regarder la télévision, en mangeant des friandises ou en buvant de l'alcool ${ }^{11} »$. Wiktionary indique l'origine suivante et ajoute les deux mots suivants « dérivés » de couch potato : desk potato et mouse potato : "From couch + potato; coined by Tom Iacino in 1976 as a pun on boob tuber (from boob tube ("television") and tuber ("potato")); trademarked by Robert Armstrong from 1976-91" ".

110 occurrences pertinentes ont été relevées en tout : 47 de couch potato et 63 de couch potatoes, (toujours en deux mots et jamais soudés) entre le 09.01. 1989 et le 11. 05.2019, sur plus de 31 ans donc, ce qui représente une moyenne d'un peu moins de 4 par an. Le nombre des occurrences se répartit ainsi :

La diffusion est lente au début (10 dans les 7 premières années : 1989-1995), avant un petit plateau de 6 années (1996-2001) avec 30 occurrences, puis des dents de scie : 7 en trois ans (2002-2004) avant 17 en deux ans (2005-2006) et nouvelle chute 11 en 5 ans (2007-2011) puis un nouveau pic de 3 ans (2012-2014) avec 17 occurrences et nouvelles petites dents de scie ensuite, entre 2 et 5 selon les années. Sauf au début, il n'y

\footnotetext{
fr.wiktionary.org/wiki/couch_potato, consulté le 26. 03. 2021.

en.wiktionary.org/wiki/couch_potato, consulté le 26. 03.2021.
} 


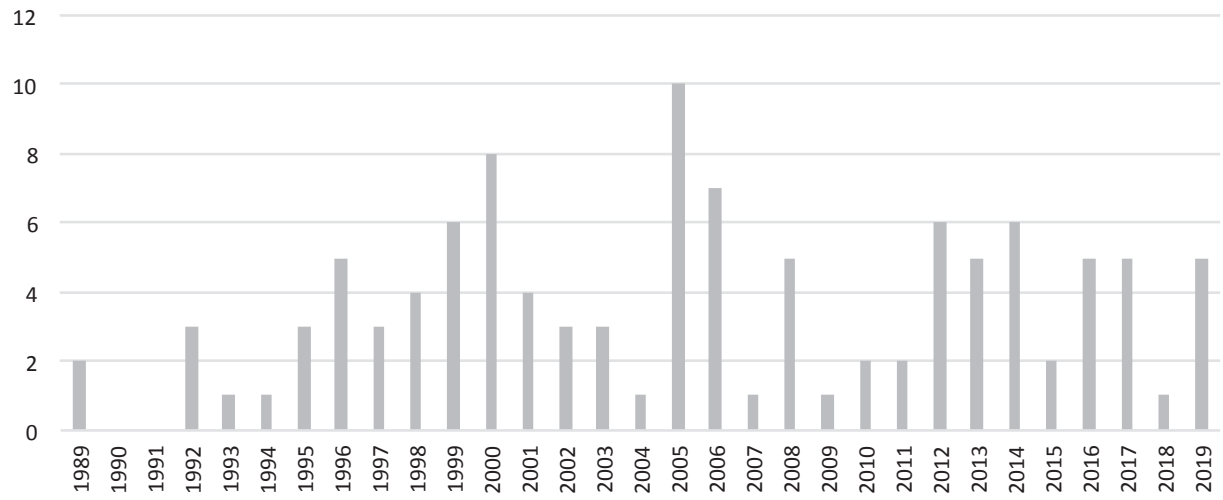

Graphique 2 : Les attestations de couch potato par année dans Europresse

a pas d'années sans occurrences. Il est difficile de rendre compte de ces variations, qui sont sans doute peu significatives.

Ces 110 occurrences sont relevées dans un grand nombre de sources de presse différentes, ce qui montre une large diffusion : Le Monde, Libération, L'Express, AFP infos françaises, AFP infos mondiales, Le journal de Saône-et-Loire, Ouest-France, Le Point, La Voix du nord, Le Figaro, Les Échos, Télérama, L'Équipe, L'Argus de l'assurance, TV mag au singulier et aussi, pour le pluriel, dans La Croix, Sud-Ouest, L'Humanité, Le Progrès (Lyon), Le Parisien, Courrier international, La Voix du Nord, L'Indépendant, Le Bien public, Le Télégramme (Bretagne).

À titre de précision, la première attestation de ce composé apparaît dans Le Monde le 9 janvier 1989, puis $2^{\text {ème }}$ le 16 novembre 1992 et $3^{\text {ème }}$ le 13 janvier 1997, toujours dans les pages du même journal pour le singulier :

(1fr) Êtes-vous un couch potato (une patate de canapé) ? Les Américains appellent ainsi le téléspectateur amorphe, affalé devant son petit [écran].

$1^{\text {ère }}$ attestation du 9/1/1989 dans Le Monde, puis $2^{\text {ème }}$ le 16/11/92 et $3^{\text {ème. }} 13 / 1 / 1997$ toujours dans Le Monde pour le singulier.

Des traductions diverses accompagnent cet emprunt : patate de canapé; drogué de télévision; traductions signifiant littéralement " pomme de terre sur canapé »; (pomme de terre sur canapé), pour le singulier et au pluriel : les avachis du divan; pommes de terre de canapé ; patates couchées ; patates de canapé ; pommes terre <sic> sur le canapé; littéralement patates sur canapé; abrutis de télé.

Patate(s) de canapé : 44 (47-3 répétitions) du 26. 01. 1988 au 11. 05. 2019.

Parmi ces emplois, 15 le sont avec couch potato à proximité, mais 29 sans (66\%). L'équivalent fonctionne souvent seul. Cette forte proportion montre que l'équivalent s'est autonomisé et joue son rôle de remplacement de l'emprunt. 11 emplois font allusion aux 


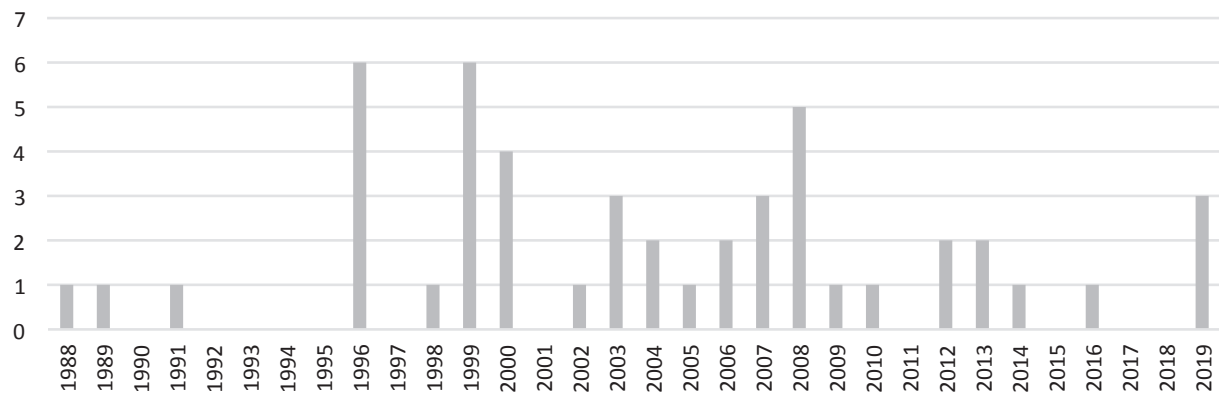

Graphique 3 : Les attestations de patate(s) de canapé par année dans Europresse

États-Unis (américains, etc.), ce qui représente un quart des emplois. Le phénomène est clairement indiqué comme étant d'origine américaine et il n'est pas impossible que les premiers emplois de couch potato soient des xénismes, dont la traduction française joue le rôle d'explication, plus que de traduction appelée à s'autonomiser et à se développer, ce qui va arriver ensuite avec l'introduction de ces comportements dans nombre de pays, en dehors de la sphère américaine. 18 emplois sont entre guillemets. Le pourcentage de $41 \%$ est assez important et indique une prise de distance de l'émetteur dans l'emploi de ce mot, pour diverses raisons, dont le caractère familier de patate.

On relève aussi, entre 1997 et 2012 et pour la même période, douze occurrences de patate couchée, trois de pomme de terre de canapé et trois de pomme de terre sur canapé. Il est remarquable à ce propos que le mot neutre pomme de terre soit moins employé que le mot familier patate, (6 contre 44 sans compter d'autres emplois de patate, v. infra), qui a l'avantage d'être plus proche de la lexie source potato. La familiarité du mot correspond bien par ailleurs à la vulgarité des personnes ainsi dénommées, qui appartiennent le plus souvent aux catégories populaires, voire défavorisées économiquement et culturellement.

D'autres formulations n'emploient pas le mot patate/pomme de terre, notamment

- abruti de télé : 3 occurrences vraiment pertinentes (un au singulier, mais pas directement lié à couch potato et huit au pluriel dont quatre fois le même énoncé et deux emplois avec télé-réalité ou télé seul). Un emploi est explicitement en lien avec couch potato (qu'il traduit, entre parenthèses)

- avachi du divan : Un seul emploi, dans Le Monde du 16/1/1989, entre parenthèses comme glose de couch potato, assimilés aux boomers, du fait de leur appétence pour la télévision.

Les emplois de ces diverses traductions sont épars et distants au début suivis de deux années de pic 2005 et 2006 qui représentent un quart des occurrences, puis emplois assez étales ensuite, avec des variations sans doute peu significatives. Un assez grand nombre de traductions (41\%) sont mises entre guillemets. Une date de création est donnée par Ouest France ${ }^{13}$, 22. 06. 2005 :

13 https://www.ouest-france.fr/, consulté le 26. 03. 2021. 
(2fr) [Les producteurs] britanniques de pommes de terre en ont assez! Ils demandent le retrait du dictionnaire de couch potato. Signifiant littéralement « pomme de terre sur canapé », cette expression apparue aux États-Unis en 1979...

soit dix ans avant la première attestation dans la presse française. Pour les équivalents, plusieurs remarques peuvent être formulées :

1. Couch est le plus souvent rendu par canapé tant dans le nombre d'expressions différentes que dans celles qui sont le plus diffusées. On trouve néanmoins une fois divan et une fois le participe passé couché, qui se veut proche du mot source couch.

2. Potato est rendu soit par patate, très proche de la source mais marqué familier en français, soit par pomme de terre, qui est la dénomination courante neutre. La ressemblance de patate avec la source potato explique que ce mot est plus fréquemment employé que pomme de terre.

3. Pommes de terre de canapé, patates couchées, patates de canapé, pommes terre <sic $>$ sur le canapé, patates sur canapé sont des calques morphologiques assez fidèles.

4. Deux traductions sont en fait assez voire fort éloignées de la source, mais elles fonctionnent néanmoins comme équivalents plus que comme explications semble-t-il, ne serait-ce que par leur brièveté : les avachis du divan (qui garde néanmoins la traduction du mot couch par divan), et abrutis de télé, qui est une synthèse néologique, équivalent sémantiquement mais qui ne doit rien, dans la forme, à la source.

Des commentaires ou gloses accompagnent souvent l'emprunt, mais le départ entre traduction et commentaire n'est pas toujours aisé à décider. L'équivalence de longueur, la proximité avec la source, une indication qu'il s'agit d'un équivalent incitent à considérer qu'il s'agit plutôt de traductions. En revanche, une longueur plus grande que l'original, due à des ajouts informatifs ou d'opinions subjectives, fait pencher la balance plutôt vers le commentaire. On a relevé : téléspectateur amorphe, affalé devant son petit [écran]...; téléphage américain; personnes passant leur temps affalées devant la télévision; ceux qui passent de longues heures affalés devant la télévision, vautrés sur leur [canapé]...; effondré sur son canapé; (vissée devant son écran de télé); affalés sur le canapé devant la télé ; personnes qui passent leur vie dans un canapé à regarder...; (le téléspectateur passif) ; individu affalé sur son canapé qui s'empiffre de chips pendant des heures devant le téléviseur; (NDLR: sédentaire); (vautré sur son canapé à regarder la télévision); téléspectateurs affalés devant l'écran. Ces données diverses appellent quelques commentaires :

1. 50 de ces 110 emplois relevés sont mis entre guillemets, ce qui représente un peu moins de la moitié (41\%), ce qui est loin d'être négligeable. Il y en a 17 (moins de la moitié) pour le singulier et 33 pour le pluriel. On les trouve tout au long de la période.

2. La plupart des commentaires portent sur des individus relevant d'une classe sociale (au sens large) et sont formulés au pluriel, en tant qu'il s'agit d'une catégorie de personnes. On indique néanmoins ci-après la forme lemmatisée, au singulier.

3. Certains mots-têtes sont neutres (ceux qui, personne, téléspectateur...) et s'opposent à des dénominations critiques, dévalorisantes (individu, type, mollusque, adolescent attardé...) ou qui sont une essentialisation d'un défaut, d'une caractéristique négative par nominalisation d'un participe ou adjectif (les affalés, avachis, abrutis...v. infra postures et traits psychologiques). 
4. Un équivalent de couch est éventuellement présent : canapé (dans six contextes), sofa, divan, fauteuil. On observe une variété plus grande que dans les traductions, mais canapé l'emporte dans tous les cas.

5. En revanche, les mots patate ou pomme de terre n'apparaissent pas. Mais apparaissent, au lieu de ces végétaux, des animaux, sous une forme dévalorisante : bêtes, mollusques, etc.

6. On note l'insertion fréquente de mots indiquant l'objet de l'activité : écran est présent sous diverses formes : petit écran (quatre fois), écran de télé ou d'ordinateur, écran de télévision. Des mots comprenant télé (avec ou sans association avec écran) sont également bien représentés : télé (deux fois), télévision (trois fois), téléviseur. On relève aussi des quasi-synonymes : poste, console. Une fois c'est le nom d'une chaîne qui est indiqué (MTV). Quant aux personnes, concernées, il s'agit de téléspectateurs (trois fois) voire de téléphages (fractocomposé hybride néologique).

7. Les indications de postures corporelles sont fréquentes et toujours dévalorisantes, qu'elles apparaissent comme mots-têtes ou comme modifieurs caractérisant d'un mot-tête : affalé (quatre fois), amorphe, avachi, vautré (trois fois) avec la variante qui se vautrent. Le groupe prépositionnel en pantoufle, est aussi un bon indicateur d'une certaine attitude casanière et peu active.

8. Des traits psychologiques et intellectuels sont également utilisés : abruti, maniaque, insensible, (téléspectateur) moyen, fan.

9. On observe une opposition entre de longues formulations purement descriptives, parfois appuyées sur des éléments de la source et des formulations lapidaires très acerbes, purement subjectives et sans lien direct avec l'emprunt source, qui sont autant de portraits charges. Relèvent de la première catégorie : ceux qui passent leur temps devant la télé, [ceux] qui passent leur vie devant les images qui défilent sur leur petit écran, personnes rivées à leur canapé devant la télé; ceux qui passent leur temps devant la télé. Dans la deuxième catégorie, on peut citer rivés à leur console, fasciné par le maximum de daube. Mais il arrive parfois que la formulation comporte des ajouts assez gratuits mais très significatifs comme la nourriture ingérée pendant ces longues séances de visionnage : gros pleins de chips (qui détourne gros plein de soupe, présent dans la BD de Tintin, dans la bouche d'un perroquet) ou fans de sport vautrés sur le sofa, la main dans un sac de chips.

10. Le mot chips est, au moins une fois, mis en relation avec potato, "pomme de terre" à partir desquelles sont confectionnés les chips. Pourtant, cela ne paraît pas devoir être l'explication de l'expression source. L'étymologie de couch potato étant peu transparente, l'expression fait plutôt songer à la masse amorphe et immobile d'un volumineux sac de pomme de terre.

\subsection{2 couch potato en polonais}

En ce qui concerne le polonais, cet emprunt est premièrement plus récent qu'en français et, par conséquent, beaucoup moins répandu. On relève uniquement deux occurrences de couch potato dans Frazeo, les deux de la presse en ligne. Il semble que le premier emploi date de l'année 2012. Après l'an 2015, il est possible de noter une légère augmentation de l'utilisation de ce composé : le corpus Polish Web 2012 de Sketch Engine 
en affiche 61 résultats, tandis que le corpus postérieur, Timestamped JSI 2014-2020, seulement trois. Bien que la plupart de ces attestations soient associées à la signification de l'emprunt anglais, il est aussi intéressant de voir apparaître de nouveaux contextes : dans l'un des extraits, on parle de finansowe couch potato, du couch potato financier. L'emprunt couch potato devient ici synonyme de paresse d'un côté, de l'autre, fait plutôt allusion à l'inefficacité de la gestion financière ${ }^{14}$. La recherche sur Google s'avère un peu plus fructueuse - en y trouve 36600000 occurrences, mais il faut souligner que la plupart d'entre elles indiquent soit les pages en anglais, soit les entrées dans les dictionnaires bilingues anglais-polonais. En plus, quoique cet emprunt soit absent dans Wikipédia polonaise, il existe une courte définition de couch potato dans l'encyclopédie PWN :

\section{(4pl) Kto to jest „Couch Potato”?}

Dosłownie „ziemniak kanapowy” — określenie osoby, której głównym zajęciem jest przesiadywanie przed telewizorem połączone z pojadaniem junkfoodu i popijaniem. Wyrażenie to zostało ukute pod koniec lat osiemdziesiątych przez niejakiego Boba Armstronga z Sacramento. Założył on „Couch Potato Movement”, zrzeszający ponad 10 tys. osób, powołując się na teorię, że telewizja dostarcza idealnych, niezastąpionych doświadczeń i przeżyćc ${ }^{15}$.

[Qui est « Couch Potato »?

Littéralement " patate de canapé » - un terme utilisé pour décrire une personne dont la principale occupation est de rester devant la télévision pour manger de la malbouffe et boire. L'expression a été forgée à la fin des années 1980 par un Bob Armstrong, de Sacramento. Suivant la théorie selon laquelle la télévision offre des expériences idéales et irremplaçables, il a fondé le « Couch Potato Movement » associant plus de 10000 personnes.]

Il reste à noter que tous les emplois de ce composé relevés dans la presse ou sur Internet sont associés au même sujet, à savoir l'attitude ou le comportement de ceux qui privilégient un mode de vie sédentaire et passif.

Quant à la traduction littérale en polonais - ziemniak kanapowy - elle est le plus souvent mise entre guillemets. Elle est aussi parfois accompagnée d'un commentaire ou d'une glose. Son emploi se limite généralement à la blogosphère - la recherche sur Google affiche 672 résultats pour ziemniak kanapowy au singulier et 17800 résultats pour ziemniaki kanapowe au pluriel, le corpus de 2012 dans Sketch Engine en propose sept et Frazeo un seul. Ce qui paraît intéressant c'est le fait que le pluriel n'est utilisé que pour la traduction en polonais. Le pluriel de l'emprunt à l'anglais - couch potatoes - n'est pas du tout attesté.

Quant aux équivalents polonais, ils sont formés habituellement sur la base du premier élément de l'emprunt, le mot couch. Le premier terme - kanapowiec ('celui qui reste sur le canapé) - est beaucoup plus répandu (on relève 330000 occurrences sur Google) et, en même temps, englobe mieux l'aspect ludique du composé anglais. Le deuxième - leń kanapowy 'fainéant du canapé' - met plutôt l'accent sur les traits de caractère et/ou la manière d'être associés à la paresse. On trouve environ 16000 occurrences pour ce dernier mot sur Google.

14 http://www.podroze.kamgora.pl/tag/ekonomicznych-i-prawniczych (cité d'après le corpus Polish Web 2012 de SE; consulté le 26. 03. 2021).

15 https://encyklopedia.pwn.pl/materialy-dodatkowe/haslo/Kto-to-jest-Couch-Potato;503905.html, consulté le 26. 03. 2021. 
Initialement, la recherche dans les deux corpus de Sketch Engine, en particulier pour kanapowiec, semble un peu déroutante. On en relève 1857 résultats dans le corpus du 2012, ce qui constitue un nombre énorme, en revanche l'analyse de ces données montre que la plupart des contextes proposés sont liés aux animaux - chiens ou chats du canapé, c'est-à-dire des animaux domestiques. Le corpus postérieur, celui de 2014-2020, filtre les informations plus efficacement : les 127 résultats affichés restent en relation avec la signification de l'emprunt.

Il convient également d'ajouter à cette liste trois formulations fort intéressantes. Premièrement, il s'agit d'un hapax sofa-man qui a apparu dans le titre d'un article de blog en $2015^{16}$, mais qui n'a pas du tout gagné en popularité. Ensuite, d'un autre hapax potatriots couch - la création et le phénomène très récents qui ont émergé en relation avec COVID-19, dans le cadre de la campagne publicitaire réalisée par Burger King aux ÉtatsUnis. La marque a forgé ce nouveau terme pour ses clients en indiquant que leur responsabilité civique est maintenant de rester à la maison sur le canapé et de manger du fast food ${ }^{17}$. En polonais, ce composé a apparu dans un article décrivant la campagne publicitaire mentionnée ${ }^{18}$. Finalement, il s'agit du mot couchersizing (couch + exercising) pour lequel on relève 599 occurrences sur Google polonais et qui désigne une sorte d'exercices ou d'activités physiques que les couch potatoes peuvent pratiquer sur le canapé.

\section{Les petites séries}

Bien moins répandus dans les deux langues que couchsurfing et couch potato, couch gag et couch promotion sont néanmoins relativement bien attestés dans la presse, surtout française.

\section{1 couch gag}

Ce composé est toujours relevé en relation avec les Simpson (en particulier dans une parodie française). Wikipédia française a une entrée « Liste des gags du canapé des Simpson », qui comporte plusieurs centaines de blagues.

Dans les archives d'Europresse, on constate sept occurrences du 12. 10. 2010 au 08. 08. 2019 (aucun couch gag n'apparaît dans le $8^{\text {ème }}$ article indiqué). On relève une occurrence en 2010, une en 2014, une en 2015, trois en 2016 et une en 2019. Quatre emplois sont mis entre guillemets, ce qui représente plus de la moitié (57,15\%). La traduction gag du canapé est donné dans les deux premiers emplois (entre parenthèses ou entre guillemets) et deux fois c'est qualifié par célèbre et fameux.

On trouve cinq emplois de gag de canapé, entre 2010 et 2016, tous en relation avec les Simpson, dont deux seulement associés à couch gag et trois non. Ces trois emplois

16 https://kamilalila.wordpress.com/2015/11/26/sofa-man-czyli-dlaczego-warto-omijac-ziemniaki -kanapowe/, consulté le 26. 03. 2021.

17 https://www.campaignlive.co.uk/article/burger-king-salutes-frontline-heroes-couch-potatriots /1679831. https://musebycl.io/advertising/burger-king-salutes-couch-potatriots-stay-home-whopper -ad, consultés le 26. 03. 2021.

$18 \mathrm{https} / /$ www.wirtualnemedia.pl/artykul/burger-kinga-stay-home-of-the-whopper-kampania-koronawirus, consulté le 26. 03. 2021. 
sont entre guillemets et deux fois accompagnés de qualificatifs : célèbre et fameux. C'est sans doute cette célébrité qui explique la grande proportion d'emplois entre guillemets, comme citations de quelque chose de bien connu sous ce nom.

(3fr) Sylvain Chomet a eu la chance de réaliser un « couch gag " (gag de canapé) pour le dessin animé américain culte «Les Simpson ». Au fil des saisons, les génériques des « Simpson » continuent à nous surprendre grâce au fameux « couch gag » ou gag de canapé : un gag nouveau pour introduire chaque nouvel épisode, au moment où toute la famille Simpson s'assoit dans le canapé. 2014/3/07

Google : 11100 résultats le 14. 05. 2020 dont :

(4fr) Le gag du canapé clôture la séquence d'ouverture des épisodes de la série [des Simpson]. Les membres de la famille se rassemblent sur le canapé pour regarder l'épisode. Il est basé sur le comique de répétition et présente les situations les plus diverses et rocambolesques ${ }^{19}$.

(5fr) Le « couch gag », séquence dans laquelle les Simpson se réunissent pour regarder l'épisode à la fin de chaque générique d'introduction de la série de Matt Groening, a 28 ans. 28 ans qui ont été résumés en une vidéo. Les " couch gags ", qui ont donc été instaurés au lancement de la série en 1989, s'enchaînent à toute vitesse au son de « Get Innocuous ! » de LCD Soundsystem dans cette vidéo. On y observe au passage l'évolution artistique de la série ${ }^{20}$.

En polonais également, ce composé fonctionne uniquement en lien avec la série The Simpsons. On ne relève qu'une occurrence dans la presse généraliste publiée le 27.04.2014 sur le site de Gazeta Wyborcza:

(5pl) Michał Socha, autor nagradzanych krótkometrażowych filmów animowanych, wyreżyserował tzw. couch gag dla niedzielnego odcinka "Simpsonów”. „To najbardziej twórcza czołówka dotychczas" - zachwyca się branżowy portal Cartoon Brew.

Couch gag to żartobliwa, zmieniająca się z odcinka na odcinek animacja, która kończy się zawsze obrazkiem rodziny Simpsonów na kanapie. Do jej stworzenia zapraszani są tylko najlepsi animatorzy, graficy czy ilustratorzy, wśród nich - Banksy, Guillermo del Toro, Bill Plympton, Sylvain Chomet i John Kricfalusi ${ }^{21}$.

[Michał Socha, auteur de courts métrages d'animation primés, a réalisé le soi-disant couch gag pour l'épisode de dimanche de la série des Simpson. «C'est l'ouverture la plus créative à ce jour ", s'enthousiasme le site web de l'industrie Cartoon Brew.

Couch gag est une animation ludique qui change d'épisode en épisode et qui se termine toujours par une image de la famille Simpson réunie sur le canapé. Seuls les meilleurs animateurs, graphistes et illustrateurs, dont Banksy, Guillermo del Toro, Bill Plympton, Sylvain Chomet et John Kricfalusi sont invités à la créer.]

19 https://fr.wikipedia.org/wiki/Liste_des_gags_du_canap\%C3\%A9_des_Simpson, consulté le 26. 03. 2021.

20 http://rockyrama.com/channel/television/Simpson/7620869/28-ans-de-couch-gag-dans-les-simpson, consulté le 26. 03. 2021.

21 https://wyborcza.pl/1,75410,15867538,Polak_stworzyl_swietna_czolowke_do_kultowych_Simpsonow _.html, consulté le 26. 03. 2021. 
En plus, on relève environ 32300 occurrences de ce composé sur Google polonais, notamment sur les blogs, les fandoms (comme Simpsons Wiki) ou sites liés à l'animation, au cinéma et au divertissement. Cependant, la plupart renvoient aux pages en anglais. De la même manière, les résultats trouvés dans les deux corpus du Sketch Engine (15 dans le corpus du 2012 et un dans le corpus 2014-2020) restent liés à la presse en ligne plutôt informelle ou à la blogosphère.

Il est aussi intéressant de noter qu'il existe quelques emplois de la traduction littérale du mot en polonais : kanapowy gag " gag du canapé » - 8540 occurrences sur le moteur de recherche Google. Tous ces résultats étant associés à la série des Simpson :

(6pl) 9-go listopada na antenie Fox pojawi się nowy odcinek „The Simpsons” zatytułowany „Simpsorama”. Będzie to crossover z bohaterami „Futuramy”, innego serialu animowanego anulowanego rok temu. Fani obydwu produkcji już od roku są kuszeni możliwością spotkania rodzinki Homera i przyjaciół Fry’a na jednym ekranie. Dzisiaj mamy możliwość rzucić okiem na słynny kanapowy gag w openingu „The Simpsons"22.

[Le 9 novembre, un nouvel épisode des Simpson, intitulé « Simpsorama » sera diffusé sur Fox. Il s'agira d'un crossover avec les protagonistes de « Futurama », une autre série animée annulée il y a un an. Les fans de deux productions sont tentés depuis un an par la possibilité de retrouver sur un même écran la famille d'Homère et les amis de Fry. Aujourd'hui, nous avons l'occasion de nous pencher sur le fameux gag du canapé dans l'ouverture des Simpson.]

\section{2 casting couch}

Casting couch correspond en français à une expression promotion canapé, défini ainsi par Wikipédia : " expression familière désignant un avantage (avancement, promotion, embauche) qu'une personne obtient en consentant à avoir des relations sexuelles avec une personne ayant le pouvoir de lui attribuer cet avantage ${ }^{23} »$.

On relève sept occurrences du 12. 05. 2007 au 04. 03. 2018, avec un saut entre 2007 et 2017 :

(6fr) Les Dessous pervers de Bollywood enquête sur le « casting couch », ou « distribution canapé ", équivalent de notre bonne vieille promotion canapé adaptée à l'univers impitoyable... ${ }^{24}$

(7fr) ... nombre de jeunes actrices doivent payer de leur personne. On appelle ça la « casting couch $»^{25}$.

(8fr) ... tellement répandue dans le milieu du cinéma qu'elle a donné lieu à la locution " casting couch », [un équivalent de notre " promotion canapé" »] ? ${ }^{26}$

(9fr) Harvey Weinstein est réapparu sur Hollywood Boulevard, mais de façon détournée. L'ex-magnat du cinéma, sous le coup de plusieurs enquêtes pour viols, harcèlement et agres-

\footnotetext{
22 https://www.spidersweb.pl/rozrywka/2014/11/07/simpsorama-coraz-blizej-zobaczcie-kanapowy-gag -crossoveru-futuramy-the-simpsons/, consulté le 26. 03. 2021.

3 https://fr.wikipedia.org/wiki/Promotion_canap\%C3\%A9, consulté le 26. 03. 2021.

4 https://www.telerama.fr/television/6565-dimanche_est_tandoori.php, consulté le 26. 03. 2021.

Aujourd'hui en France 2007/5/13, Tout n'est pas rose à Bollywood.

6 Courrier international 19. 10. 2017, Harvey Weinstein ne peut laver tous les péchés d'Hollywood-USA Today McLean (Virginie).
} 
sions sexuelles, est revenu jeudi sous la forme d'une statue à taille réelle. Elle représente le producteur américain en peignoir sur un canapé. L’œuvre, signée par les artistes Plastic Jesus et Joshua Ginger Monroe, s'intitule « Casting Couch » ( Promotion canapé ») et est située près du Dolby Theater, où se tiendra cette nuit la cérémonie des Oscars ${ }^{27}$.

On relève deux occurrences en 2007 à propos d'un film indien, avec guillemets, traduction et commentaires, comme si c'était un xénisme alors que la chose et un mot existent en français. Est-ce pour faire couleur locale? Puis, on observe un trou de 10 ans et cinq occurrences en deux ans (2017 et 2018), toutes liées à l'affaire Weinstein. Trois de ces dernières occurrences sont un nom propre d'œuvre (une sculpture représentant Weinstein, producteur de cinéma condamné depuis pour viol de jeunes actrices). L'équivalent promotion canapé est quasiment toujours présent.

En ce qui concerne la langue polonaise, il semble que ce composé soit presque complètement absent. Les seules occurrences sur Google renvoient soit au titre de trois films et deux livres soit au nom du groupe de hard métal et de rock polonais. Cependant, on en note une seule occurrence dans les deux corpus de Sketch Engine choisi pour cette étude, il se peut donc que ce terme commence à s'installer petit à petit aussi en polonais.

Dans les deux composés de ces petites séries, on remarque l'absence de foisonnement néologique aussi bien en français qu'en polonais si ce n'est que casting couch, qui a un équivalent français bien antérieur et bien connu (promotion canapé), reçoit dans sa première occurrence une traduction littérale, plus proche de l'original : distribution canapé, où distribution est la traduction de casting, mais, d'une part, audition conviendrait peutêtre mieux et, d'autre part, ce calque est peu naturel du fait de l'existence en français d'une dénomination exactement équivalente. Quant au composé précédent, couch gag, il n'a qu'une seule traduction, gag de canapé, pas toujours associée à l'emprunt, et qui s'est autonomisée puisqu' on la trouve parfois sans l'emprunt, mais toujours en relation avec le film d'animation bien connu Les Simpson. Quant au polonais, il n'existe qu' une seule traduction pour l'un des composés en question : kanapowy gag pour couch gag.

\section{Hapax et emprunts de faible diffusion}

Les hapax ou quasi-hapax, inévitablement nombreux, ne renseignent pas sur la lexicalisation, mais comportent souvent des traductions et des gloses intéressantes.

\subsection{Les hapax dans la presse}

\subsection{1 couch air car}

Pour ce premier emprunt, on ne trouve qu'une seule occurrence dans les archives d'Europresse :

Aujourd'hui en France, Édition Principale, Guide dimanche, dimanche 04. 03. 2018. 
(10fr) ... flemme de conduire pour rentrer. Aucun problème ! Des dizaines de marques ont déjà lancé le couch air car (le matelas gonflable pour voiture). Il fonctionne comme un matelas gonflable classique ${ }^{28}$.

L'emprunt, qui n'a pas de marque typographique, est traduit dans une parenthèse. Il est étonnant de ne trouver qu'une seule occurrence dans la presse pour une invention développée par une multitude de marques. Les pages françaises de Google consultées le 14. 05. 2020 pour couch air indiquent 4260 résultats, dont les premiers ne concernent pas spécifiquement des matelas gonflables pour voitures, mais des matelas gonflables en général. On trouve cependant Couch Air comme nom d'une marque qui commercialise ce type de dispositif pour des voitures. La requête « couch air car» dans tous les contenus ne fournit que six résultats dont un seul en français.

Bien que ce mot soit complètement absent de la presse polonaise (0 occurrences dans la presse généraliste, dans les deux corpus polonais de Sketch Engine analysés et dans Frazeo), il est relativement bien représenté en ligne (137 000000 résultats sur Google, dont cependant beaucoup en anglais) et indique, comme en français, les matelas ou les sofas gonflables, pas nécessairement pour voitures. Il existe aussi quelques équivalents ou quasi-équivalents concernant le même type de produits qui circulent dans les pages virtuelles polonaises : air sofa, lazy sofa, lazy bag. On peut également trouver des traductions directes en polonais : powietrzna sofa (environ 208000 résultats sur Google). Cependant, il serait difficile, voire impossible, de déterminer laquelle de ces formes est dominante.

\subsection{2 couch battle}

En ce qui concerne la recherche dans Europresse pour cet emprunt, on ne relève aucun résultat en français de France (mais quatre en anglais dans requête " tous les contenus »). En revanche, le moteur Google atteste 107 résultats (ce qui est peu) le 14. 05. 2020 dont beaucoup de pages en anglais, un nom propre de jeu, et des sites vendant des vêtements, etc.

On retrouve ce composé dans La Liberté, 11. 08. 2016 :

(11fr) Cela pourrait s'appeler « couch battle». La bataille pour un canapé. Une joyeuse compétition qui a lieu chaque soir durant l'Open Air Cinéma. Le principe ? Faire preuve de créativité pour pouvoir regarder le film assis sur un divan.

« Il y a 21 places à gagner. Les vainqueurs peuvent ensuite s'y installer pour visionner le film », résume Gaëtan Portmann, l'un des fondateurs, il y a 17 ans, du Movie Bar de l'Open Air Cinéma. C'est là que se déroule le concours mis en place il y a environ dix ans. "Au début, les gens se disputaient les canapés. Nous avons alors décidé de faire gagner les places », ajoute cet organisateur de soirées connu également sous le nom de DJ Goton Le Cool.

Il semble bien que, dans ce sens, ce soit un hapax de type allogénique, qui ne s'est pas répandu au-delà du cercle de ce cinéma particulier. Comme le mot battle s'est largement diffusé en français, il y a quelques années déjà pour divers types d'affrontements ou de

28 https://www.charentelibre.fr/, consulté le 31. 07. 2018. 
compétitions, il n'est pas étonnant que ce mot ait été employé, hors de la sphère anglophone, accolé à couch pour une compétition dont l'enjeu est une place confortable sur un canapé. Il est remarquable que l'acte de nomination ( «s'appeler») soit introduit pas par le verbe pouvoir au conditionnel, ce qui renforce l'hypothèse d'un allogénisme. Cependant, ce mot est absent en polonais - il n'est présent dans aucun corpus ni moteur de recherche choisi pour cette étude.

\subsection{3 couch commander}

Pour cet emprunt, Europresse affiche une seule occurrence sur le site de Stratégies $\mathrm{n}^{\circ}$ 1817 du 11. 06. 2015, dans l'article La gloire à la portée des flemmards :

(12fr) ...ce bracelet, relié à une application, permet de connaître son niveau de flemmardise, du niveau « Couch commander» à « Cryogenic! " pour les plus léthargiques. Un moyen de promouvoir la marque Joe Boxer, associée...

S'il y a, dans cet article, des guillemets de citation, il n'y a ni traduction ni explication.

Le moteur de recherche Google livre 758 résultats le 14. 05. 2020, dont la plupart en lien avec une vidéo, de mai 2016, où Barack Obama s'autoparodie en " commandant en chef du canapé ». Mais on trouve quelques occurrences antérieures, de 2008 par exemple, qui semblent montrer que ce composé circule en anglais (américain ?) et que la vidéo de 2016 a contribué à diffuser largement. En revanche, il ne semble pas que ce soit importé en français, où ce composé pourrait peut-être relever du xénisme. On trouve sur M6, 02. 11 . 2015 une allusion plus explicite à l'application de la mesure de l'inactivité :

(13fr) L’appli suit le temps passé sans bouger (de votre canapé par exemple) et vous décerne des «titres honorifiques » au fur et à mesure que vous prouvez vos efforts : « Couch Commander », « Cryogenic » ou encore « You Rock! ».

En ce qui concerne la langue polonaise, on note uniquement 11 occurrences de ce composé, dont deux dans le Timestamped JSI web corpus 2014-2020 pour le polonais, et les autres sur Google, toutes font référence à un film parodique de Barack Obama.

\subsection{4 couch compagnie}

Europresse livre une occurrence pour ce composé :

(14fr) Le 1er avril 2008, Frédéric Diaw immatricule sa société et sa marque TshOtsch à Quimper. "Au début, c'était une couch compagnie, installée dans mon appartement. Je travaillais sur mon canapé ! Pendant les 4 premières années. ${ }^{29} "$

En revanche, 1800 résultats sont affichés par le moteur de recherches Google, très souvent comme un nom propre d'une entreprise de meubles. Dans l'attestation ci-dessus, le composé ressemble à un allogénisme (anglicisme sans modèle attesté en anglais) ou

29 https://www.ouest-france.fr/bretagne/quimperle-29300/tshotsch-la-marque-qui-surfe-jusqua -letranger-3396611, consulté le 31. 07. 2018. 
un hybride, du fait de l'orthographe française de compagnie. Bien que la requête ait porté exclusivement sur les pages françaises, nombre de résultats sont des énoncés en anglais. Dans le cas de la langue polonaise, seulement des énoncés en anglais apparaissent, ce qui permet de constater que ce terme n'est pas du tout utilisé en polonais.

\subsection{5 couch dances}

Europresse : 1 en français et 2 en anglais (dans tous les contenus)

(16fr) L'actrice porno Stormy Daniels, la bombe à retardement de Donald Trump

La règle en revanche ne dit rien des couch dances, ces séances à quinze dollars au cours desquelles les mêmes performeuses, en string cette fois se frottent en cadence à des clients calés au fond d'une banquette ${ }^{30}$.

Google : 2170 résultats le 14. 05. 2020 pour les pages françaises, 867000 résultats pour les pages polonaises, dont beaucoup - en anglais malgré la requête - de renvois à des sites pour visionner des images de danseurs et danseuses sur des canapés, ce qui ne correspond pas à l'acception de la seule occurrence trouvée dans la presse française, qui semble relever du xénisme plus que de l'emprunt, même s'il n'y a pas de marques typographiques, mais il y a une glose explicative détaillée. Tous les résultats trouvés sur les pages polonaises correspondent aux collections de photos mentionnées ci-dessus. On ne trouve aucune occurrence dans la presse polonaise généraliste.

\subsection{6 couch detective}

Europresse : 1 seule occurrence sur le site de TV Mag (site web), du 10. 9. 2014 :

(17fr) Baptisé Couch Detective (littéralement : Détective sur canapé), il est décrit comme une comédie policière mystérieuse, irrévérencieuse, construite sur... ${ }^{31}$

Cette occurrence est un nom propre, avec majuscule, introduit par un terme indiquant l'acte de nomination (baptisé). Il n'est pas accompagné de guillemets mais est traduit dans une parenthèse avec la précision « littéralement » qui se présente comme une traduction mot à mot. Google donne 575 résultats le 14.05. 2020. On y retrouve en tête l'occurrence citée ci-dessus, tirée du Figaro (TVmag), puis des sites permettant d'accéder à des photos ou extraits de cette série, ainsi qu'à des produits dérivés. Beaucoup sont en anglais, malgré la requête restreinte « dans les pages en français ».

En polonais, on ne trouve aucun résultat pour ce mot dans la presse généraliste. Cependant, il circule dans les articles en ligne où il apparaît en lien avec le titre de série télévisée déjà mentionnée. Il est difficile de fournir les données numériques, vu que la plupart des occurrences sont en anglais.

30 https://www.lemonde.fr/m-actu/article/2018/04/13/stormy-daniels-la-bombe-a-retardement-de -donald-trump_5285141_4497186.html, consulté le 31.07.2018.

31 https://tvmag.lefigaro.fr/programme-tv/article/serie/83746/couch-detective-la-fin-des-series -policieres-glauques-.html, consulté le 31. 07. 2018. 


\subsection{7 couch news}

Europresse : 1 occurrence (répétée à la même source), Sud-Ouest, Toutes éditions, mercredi 31. 08. 2016 :

(18fr) La chaîne d'info publique n'a d'ailleurs pas le monopole du canapé, très en vogue en cette rentrée sur les plateaux télé, au point que l'on parle de « couch news » ${ }^{32}(1)$.

(1) «L’info canapé », en bon français

On note l'emploi des guillemets et surtout la traduction en note infrapaginale (à propos des chaînes d'information en continu)

Google : 1340 résultats le 14. 05. 2020, dont plusieurs sont liés à la création de la chaîne d'information publique en continu, sur le canal 27 de la TNT début septembre 2016, et de la nouvelle formule de L :

(19fr) Cette tendance, c'est le « couch news ». En gros, "l'info sur canapé ». Un dispositif qui nous vient des États-Unis où les chaînes info ont pris l'habitude de présenter certaines chroniques ou interviews de leurs matinales sur des canapés ${ }^{33}$.

(20fr) Sur la forme, un habillage très largement inspiré de celui des journaux de TF1 et puis quelques petits détails. Par exemple, à $6 \mathrm{~h} 34$, ce matin, on a pu notamment découvrir ces fameux canapés pour mener certaines interviews. Une tendance venue des États-Unis, le « couch news », pour créer une plus grande convivialité ${ }^{34}$.

Cependant, il semble que ce mot soit complètement absent de la presse (généraliste ou autre) polonaise. Il ne circule pas non plus dans la blogosphère ou sites Internet polonais.

Sur ces sept composés en couch attestés une seule fois dans la presse française (ou québécoise pour un d'entre eux), quatre sont accompagnés d'une traduction (unique) identifiée explicitement comme telle ou non, deux reçoivent des explications plus ou moins précises, et un est laissé complètement à l'interprétation du récepteur, sans traduction ni explication précise : la connaissance de l'anglais et le contexte sont les seuls recours pour construire le sens. Mais, ces composés n'apportent pas beaucoup de données exploitables pour le thème retenu dans cette étude.

\subsection{Les curieusement absents de la presse}

Un composé relevé dans la première requête de couch (couch gay) n'apparaît pas quand on fait une requête sur lui. S'y ajoute un autre composé trouvé ailleurs (couch coop platformer). On en trouve néanmoins des attestations à l'aide du moteur de recherche Google.

32 https://www.sudouest.fr/, consulté le 31. 07. 2018.

$33 \mathrm{https} / /$ wwww.europe1.fr/emissions/Le-fait-medias-du-jour/france-info-les-premiers-essais-de-la -chaine-dinfo-publique-diffuses-sur-youtube-2829631, consulté le 31. 07. 2018.

34 https://www.europe1.fr/emissions/Le-fait-medias-du-jour/lci-la-chaine-dinfo-devoile-son-nouveau -visage-2832319, consulté le 31. 07. 2018. 


\subsection{1 couch gay}

Europresse (France) : 0 occurrence

Google : Ce ne sont pas moins de 20200 résultats qui sont affichés par Google, renvoyant tous, si l'on se fie, pour les premières pages, aux deux ou trois lignes qui apparaissent pour chaque résultat, à des sites pornographiques homosexuels masculins.

En ce qui concerne la langue polonaise, on constate les mêmes résultats : 0 occurrence dans la presse généraliste, 0 occurrence dans les deux corpus de Sketch Engine ou Frazeo. La recherche sur Google s'avère aussi inefficace : comme en français, tous les résultats renvoient à des sites pornographiques homosexuels masculins, dont très peu en polonais.

\subsection{2 couch coop platformer}

Europresse : 0 en français de France, mais un dans tous les contenus, celui relevé ci-dessous, au Québec.

Google : trois résultats seulement dont deux en anglais et le troisième, en français, dans Le Soleil, journal québécois, à propos d'un jeu en ligne nommé Chariot.

(21fr) Dans le jargon des gamers, Chariot est un couch coop platformer. « Puisque les deux personnages, la jolie princesse et son vaillant fiancé, sont liés par un câble l'un à l'autre, les deux joueurs doivent constamment coopérer pour permettre aux héros d'atteindre éventuellement leur but », explique Steve Couture ${ }^{35}$.

Quant au polonais, cet emprunt semble être absent. Cependant, il est intéressant de souligner qu'il existe quelques-unes pour la requête ayant une signification similaire, à savoir couch Co-Op. On relève environ 191000 résultats sur Google, deux dans les deux corpus de Sketch Engine choisis pour cette analyse et quatre dans Frazeo. Toutes ces trouvailles renvoient uniquement à des articles en ligne concernant les jeux coopératifs. Il faut également ajouter que deux traductions de cette dernière occurrence circulent en polonais : kanapowy co-op, kanapowy coop, aussi dans les articles en ligne ou les blogs thématiques :

(6pl) Kanapowy co-op to jedna z tych rzeczy, za którymi niezwykle tęsknię, bo to ten jedyny, magiczny element gier chodzący za mną od dzieciństwa oraz jeden z powodów mojego zarażenia się "gamingowym bakcylem" ${ }^{36}$.

[Le co-op du canapé est l'une des choses qui me manquent le plus, parce que c'est le seul élément magique des jeux qui m'accompagne depuis l'enfance et l'une des raisons pour lesquelles j’ai attrapé le « bug du jeu».]

Ensuite, il parait que la forme que les joueurs privilégient (environ 2810000000 résultats sur le moteur de recherche Google) se limite au deuxième segment du mot : coop ou co-op. Elle apparaît parfois déclinée : coopa, co-opem.

\footnotetext{
35 https://www.lesoleil.com/affaires/frima-lance-chariot-4f76400058d1c8595ab7e8d9bdd74133, consulté le 31. 07. 2018.

36 https://www.tabletowo.pl/gdzie-te-gry-z-kooperacja, consulté le 26. 03. 2021.
} 


\section{Stratégies de choix d'équivalents en français et en polonais}

Dans les deux langues étudiées, l'emprunt direct prédomine dans tous les cas. Mais les propositions d'équivalents ne manquent pas, même si ces derniers servent surtout de gloses explicatives; il n'est pas exclu que certains soient susceptibles de lexicalisation. Le tableau 4 ci-dessous, indique les équivalents relevés dans les analyses en 2 et 3 , ordonnés selon leur proximité du modèle de langue anglaise, soit les plus littéraux d'abord pour finir par les plus éloignés.

Tableau 4 : Équivalents relevés des composés en couch-

\begin{tabular}{|c|c|c|}
\hline Emprunt à l'anglais & Equivalent français & Equivalent polonais \\
\hline couch surfing & $\begin{array}{l}\text { surfing/surfer sur canapé } \\
\text { hébergement sur le canapé (hébergement } \\
\text { grâcieux, gratuit) } \\
\text { nuit sur canapé } \\
\text { canapé en ligne } \\
\text { tourisme sofa } \\
\text { passer d'un canapé à l'autre } \\
\text { se faire prêter (prêt de) canapé }\end{array}$ & $\begin{array}{l}\text { surfowanie po kanapach } \\
\text { surfująca kanapa }\end{array}$ \\
\hline couch potato(es) & $\begin{array}{l}\text { patate de canapé } \\
\text { pomme de terre de canapé sur (le) canapé } \\
\text { patate couchée } \\
\text { abruti de télé } \\
\text { avachi du divan }\end{array}$ & $\begin{array}{l}\text { ziemniak kanapowy } \\
\text { kanapowiec } \\
\text { leń kanapowy } \\
\text { sofa-man } \\
\text { potatriots couch }\end{array}$ \\
\hline $\begin{array}{l}\text { couch coop } \\
\text { (platformer) }\end{array}$ & & $\begin{array}{l}\text { kanapowy co-op } \\
\text { kanapowy coop }\end{array}$ \\
\hline couch air car & matelas gonflable pour voiture & $\begin{array}{l}\text { powietrzna sofa } \\
\text { air sofa, lazy sofa, lazy bag }\end{array}$ \\
\hline couch battle & bataille pour un canapé & \\
\hline Couch Detective & détective sur canapé & \\
\hline couch news & info canapé & \\
\hline couch gag & gag du canapé & kanapowy gag \\
\hline casting couch & $\begin{array}{l}\text { promotion canapé } \\
\text { distribution canapé }\end{array}$ & \\
\hline
\end{tabular}

Le cas le plus fréquent est la traduction directe, souvent mot à mot, en français et en polonais. On le constate en particulier pour les hapax où les formes peu attestées (couch news = info canapé ; gag du canapé, kanapowy gag, etc.). Cette attitude de la part du/de la journaliste peut se comprendre; pourquoi aller plus loin que de décoder l'expression anglaise ? Partant du même principe, les journalistes proposent des équivalents moins précis mais qui sont parfaitement compréhensibles en contexte (hébergement grâcieux, gratuit, plutôt qu'hébergement sur le canapé). 
C'est dans les grandes séries que l'on relève le plus de variation au niveau des équivalents dans les deux langues. Pour couch surfing, le polonais se cantonne à des équivalents assez littéraux (on se demande si surfująca kanapa n'est pas une interprétation erronée, car ce n'est pas le canapé qui surfe, mais plutôt l'hôte). Le français emploie bien surfer sur (le) canapé et surfing sur (le) canapé, mais les modulations sont nombreuses : le canapé peut se transformer en sofa ou en divan, l'hébergement en nuit, etc. On relève également des tournures peu lexicalisées (passer d'un canapé à l'autre, etc.) tout à fait appropriées pour la traduction mais peu fonctionnelles pour un lexique. L'autre série importante, couch potato, a inspiré les journalistes français et polonais. Nous avons déjà noté la préférence en français pour patate, moins formel (et plus court) que pomme de terre et plus proche de l'anglais. Certains équivalents renoncent à la métaphore pour se focaliser sur la cause (abruti de télé) ou la posture de la personne : avachi du divan. Le polonais fait preuve de créativité : à côté de la traduction directe, on relève leń kanapowy (fainéant du canapé) et surtout kanapowiec (l'agent, celui qui reste sur le canapé) ainsi que ce qui pourrait bien être un allogénisme (anglicisme créé par les Polonais) : sofaman. Ce n’est pas le seul exemple de ce type de faux emprunts, car on signale également lazy sofa, lazy bag comme équivalents de couch air car, lui-même sans doute également un allogénisme.

\section{Conclusion}

On constate une certaine constance d'emploi de couch potato et de ses équivalents, qui sont les plus anciens de cette série de composés en couch-, même s'ils sont moins abondants maintenant qu'ils ne l'ont été (peu apparaissaient dans les requêtes sur Timestamped JSI 2014-2016). Quant à couch surfing, un décalage d'une dizaine d'années est observable entre la création aux États-Unis et l'importation en français, et davantage en polonais. Dans les deux langues, couch surfing semble figurer comme xénisme d'abord, avec la mention qu'il s'agit d'une pratique typiquement américaine, avant que ce mode de vie ne franchisse les frontières et ne s'installe en Europe, ce qui fait basculer le statut du xénisme à l'emprunt. Parallèlement à ce changement de statut, un autre s'est manifesté : le passage d'un nom propre de site à un nom commun par antonomase ou déonomastiques, considéré comme une déchéance par les juristes. Ce composé a servi de modèle à toute une série de composés en couch, qui se sont pour beaucoup peu répandus, sauf la série des Simpson (couch gag). Couch potato et couch surfing, les plus répandus des emprunts en couch, ont suscité un foisonnement néologique d'équivalents dans les deux langues, aux succès divers. On note aussi un grand nombre de commentaires qui se distinguent des « traductions " par des longueurs différentes par rapport à l'original, par des précisions factuelles ajoutées et aussi par des opinions subjectives explicites ou implicites (dans le choix des mots).

En ce qui concerne les commentaires des scripteurs, on constate une corrélation entre le nombre d'équivalents proposés pour rendre le sens du mot anglais et celui des remarques qui les accompagnent. Là où les équivalents sont nombreux les commentaires le sont aussi, ce qui s'explique sans doute par le très faible degré de lexicalisation des «traductions » proposées. 


\title{
BIBLIOGRAPHIE
}

Buchi, É. (1993) : «Le traitement des déonomastiques dans le FEW». In : Hilty, Gerold (éds), Actes du XXe Congrès international de linguistique et de philologie romanes. (Zürich, 1982), Tübingen / Basel : Francke Verl., tome IV, pp. 69-78.

Bušta, J., Herman, O., Jakubíček, M., Krek, S. et Novak, B. (2017) : JSI Newsfeed Corpus. In The 9th International Corpus Linguistics Conference. Corpus Linguistics 2017 Conference, University of Birmingham,

https://www.birmingham.ac.uk/Documents/college-artslaw/corpus/conference-archives/2017/general /paper382.pdf, consulté le 26. 03. 2021.

Jacquet-Pfau, C. (2018) : « Des emprunts néologiques pour exprimer le partage », dans Jacquet-Pfau, C, Napieralski, A. et Sablayrolles, J.-F. (éds), Emprunts néologiques et équivalents autochtones : étude interlangues. Université de Lodz, pp. 177-200. https://wydawnictwo.uni.lodz.pl/produkt/emprunts-neologiques-et-equivalents -autochtones-etudes-interlangues/, consulté le 23. 03. 2021.

Steuckardt, A. et Niklas-Saliminen, A. (éds) (2005) : Les marqueurs de glose. Aix-en-Provence : Publications de l'Université de Provence.

Tonti, M. (2020) : Le nom de marque dans le discours au quotidien. Prisme lexiculturel et linguistique. Collection : laboratorio@francesisti.it, Paris : L'Harmattan.

\author{
Sitographie \\ http://www.europresse.com/ \\ https://www.sketchengine.eu/ \\ http://frazeo.pl/ \\ https://fr.wiktionary.org \\ https://en.wiktionary.org \\ https://fr.wikipedia.org \\ http://dziennikturystyczny.pl \\ http://www.kasiavictor.com/pl \\ https://www.ouest-france.fr/ \\ http://www.podroze.kamgora.pl \\ https://encyklopedia.pwn.pl \\ https://www.campaignlive.co.uk \\ https://musebyclio \\ https://kamilalila.wordpress.com \\ https://www.wirtualnemedia.pl \\ http://rockyrama.com \\ https://www.spidersweb.pl \\ https://www.telerama.fr \\ https://www.charentelibre.fr/ \\ https://www.laliberte.ch/ \\ https://www.strategies.fr/ \\ https://www.lemonde.fr \\ https://tvmag.lefigaro.fr \\ https://www.sudouest.fr/ \\ https://www.europe1.fr \\ https://www.lesoleil.com \\ https://www.tabletowo.pl \\ https://www.ethnopassion.pl
}


Jean-François Sablayrolles

UMR 7597 Histoire des théories linguistiques

Anna Bobinska

Institut d'Études Romanes, Université de Lodz

ul. Pomorska 171/173, 90-236 Lodz, PL

anna.bobinska@uni.lodz.pl

John Humbley

CLILLAC-ARP EA 3967

Université de Paris

Case 7002, 75205 Paris Cedex 13, FR

humbley@eila.univ-paris-diderot.fr 